\title{
CONVEX SETS AND SUBHARMONICITY OF THE DISTANCE FUNCTION
}

\author{
M. J. PARKER
}

(Communicated by Irwin Kra)

\begin{abstract}
In this paper we show that in order for a proper closed subset $F$ of a plane domain $D$ to be convex it is necessary and sufficient that $\operatorname{dist}(\cdot, F)$ be subharmonic in $D$. We also show that this result does not hold for higher dimensions.
\end{abstract}

1. Introduction and main results. Throughout this paper $F$ is a nonempty closed subset of $\mathbb{R}^{N}(N \geq 2)$. By $C F$ we denote the complement of $F$ in $\mathbb{R}^{N}$ and by $\partial F$ the finite boundary of $F$.

We define the distance function $d_{F}$ from $F$ by

$$
d_{F}(x)=\operatorname{dist}(x, F)=\inf _{y \in F}\|x-y\| \quad\left(x \in \mathbb{R}^{N}\right)
$$

where $\|\cdot\|$ denotes the Euclidean norm.

For a given $x$ in $\mathbf{R}^{N}$ and a positive real number $r, S(x, r)$ denotes the sphere centre $x$, radius $r$, and $B(x, r)$ denotes the open ball of centre $x$ and radius $r$. We say that $B(x, r)$ touches $F$ if $B(x, r) \cap F$ is empty and $S(x, r) \cap F$ is nonempty. Further if $B(x, r)$ touches $F$ and $y \in S(x, r) \cap F$ then we say that $B(x, r)$ touches $F$ at $y$. For a function $f$ integrable with respect to the surface area measure $\sigma$ on $S(x, r), \mathcal{M}(f, x, r)$ denotes the surface mean of $f$ over $S(x, r)$.

Given two points, $x$ and $y$, in $\mathbf{R}^{N}$ we define $\overline{x y}$ to be the (closed) line segment joining $x$ and $y$, and $x y$ to be $\overline{x y} \backslash\{x, y\}$. If $T$ is a closed convex set then we call $t \in T$ an extreme point of $T$ if $t$ is not an element of $t_{1} t_{2}$ for all points $t_{1}$ and $t_{2}$ in $T$.

Motzkin's Theorem [3, Theorem 7.8] states that a nonempty closed set $F$ in $\mathbf{R}^{N}$ is convex if and only if every point in $\mathbf{R}^{N}$ has a unique nearest point in $F$. Armitage and Kuran [1, Theorem 3] used this result to prove that $d_{F}$ is subharmonic in $\mathbf{R}^{N}$ if and only if $F$ is convex. In this paper we prove a local Motzkin-type theorem in order to obtain a local version of Armitage and Kuran's result in the case where $N=2$. We also show that our second result does not hold in higher dimensions.

The results are as follows.

THEOREM 1. Let $F$ be a nonempty proper closed subset of $\mathbf{R}^{N}(N \geq 2)$, let $t$ be a point of $\partial F$ and let $\varepsilon$ be a (strictly) positive real number.

If every point in $B(t, \varepsilon)$ has a unique nearest point in $F$ then there exists a ball of radius $\varepsilon$ which touches $F$ at $t$.

THEOREM 2. Let $F$ be a nonempty proper closed subset of $\mathbf{R}^{2}$ and let $D$ be a domain such that $F \subset D$. Then $d_{F}$ is subharmonic in $D$ if and only if $F$ is convex.

Received by the editors March 16, 1987.

1980 Mathematics Subject Classification (1985 Revision). Primary 31A05; Secondary 52A10.

Key words and phrases. Convex set, subharmonic function. 
2. Some preparatory results. We first give a fairly classical result which we do not want to call our own.

LEMMA A. Let $F$ be closed in $\mathbb{R}^{N}$ and let $B(x, r)$ be a ball touching $F$ at only one point $y_{0}$. Choose any $y$ in $\overline{B(x, r)} \backslash\left\{y_{0}\right\}$. Then there exists a (strictly) positive number $\delta$ such that

$$
\overline{B\left(x+\varepsilon\left(y-y_{0}\right), r\right)} \subset C F
$$

for all $\varepsilon$ in $(0, \delta)$.

For a sketch of the proof we note that if the lemma failed there would exist a null sequence $\left(\varepsilon_{n}\right)$ such that $(*)$ did not hold for each $\varepsilon_{n}$. This would give rise to a convergent sequence of points $\left(x_{n}\right)$ such that $x_{n} \in \overline{B\left(x+\varepsilon_{n}\left(y-y_{0}\right), r\right)} \cap F$ for each $n$. The limit $x_{0}$ of $\left(x_{n}\right)$ would be distinct from $y_{0}$ and would lie in $F$ and on $S(x, r)$ which is not possible since $y_{0}$ is the only point at which $B(x, r)$ touches $F$.

From Armitage and Kuran [1, Lemma 2] we deduce the following whose proof is left to the reader.

LEMMA B. Let $U$ and $F$ be open and closed respectively in $\mathbb{R}^{N}$. If $d_{F}$ is subharmonic in $U$ then every point in $U$ has a unique nearest point in $F$.

In the absence of any reference we shall prove the following simple result.

LEMMA 1. Let $F$ be a closed set in $\mathbb{R}^{N}$ and let $S$ be a set in $\mathbb{R}^{N}$ having the property that every point $x$ in $S$ has a unique nearest point $f(x)$ in $F$. Then $f: S \rightarrow F$ is continuous in $S$.

The proof is as follows. Take any sequence $\left(x_{n}\right)$ in $S$ such that $\left(x_{n}\right)$ converges to a point $x_{0}$ in $S$ and $\left(f\left(x_{n}\right)\right)$ converges to a point $y_{0}$ in $F$. It is enough to prove that $y_{0}=f\left(x_{0}\right)$. Now

$$
\left\|x_{0}-y_{0}\right\|=\lim _{n \rightarrow \infty}\left\|x_{n}-f\left(x_{n}\right)\right\|=\lim _{n \rightarrow \infty} d_{F}\left(x_{n}\right)=d_{F}\left(x_{0}\right)
$$

so by the uniqueness of $f\left(x_{0}\right)$ we obtain the result.

We now move on to the proofs of the theorems.

3. Proof of Theorem 1. Suppose by contradiction that there is no ball of radius $\varepsilon$ touching $F$ at $t$. Hence $d_{F}<\varepsilon$ in $\overline{B(t, \varepsilon)}$ and so, by the continuity of $d_{F}$, there exists a positive real number $\delta$ such that $d_{F}<\delta<\varepsilon$ in $\overline{B(t, \varepsilon)}$. Choose any $q \in B(t, \varepsilon-\delta) \cap C F$ and let $Q$ be the set given by

$$
Q=\left\{x \in \mathbb{R}^{N}: q \in \overline{B\left(x, d_{F}(x)\right)}\right\}=\left\{x \in \mathbb{R}^{N}: d_{F}(x) \geq\|x-q\|\right\} .
$$

Clearly $Q \subset B(q, \delta) \subset B(t, \varepsilon), Q$ is nonempty and $Q$ is compact. Therefore $d_{F}$ attains its supremum in $Q$ at a point $x_{0}$ of $Q$. By the hypothesis of the theorem $x_{0}$ has a unique nearest point in $F$ so $B\left(x_{0}, d_{F}\left(x_{0}\right)\right)$ touches $F$ at only one point, $p$ say. By Lemma A there exists an $\alpha$ in $(0,1)$ such that

$$
\overline{B\left(x_{0}+\alpha(q-p), d_{F}\left(x_{0}\right)\right)} \subset C F .
$$

Since $\alpha \in(0,1)$ we have that $q \in B\left(x_{0}+\alpha(q-p), d_{F}\left(x_{0}\right)\right)$ and therefore $x_{0}+$ $\alpha(q-p) \in Q$. By $(* *)$ we see that

$$
d_{F}\left(x_{0}+\alpha(q-p)\right)>d_{F}\left(x_{0}\right)=\sup _{x \in Q} d_{F}(x)
$$

a contradiction. 
4. Proof of Theorem 2. The "if" part of the theorem is covered by $[\mathbf{1}$, Theorem 3] so it remains to prove the "only if" part.

By Lemma B every point in $D$ has a unique nearest point in $F$ so the function $f$ of Lemma 1 is continuous in $D$. Since $D$ is a domain and $f(D)=F$ we have that $F$ is path connected. Suppose, by contradiction, that $F$ is nonconvex. By [3, Theorem 4.2] there exist points $x$ and $y$ in $F$ such that $x y \subset C F$. The path connectedness of $F$ implies that there exists a path in $F$ with no self-intersections joining $x$ and $y$ (see $[4,31.6$, p. 222]). By composing this path with $\overline{x y}$ we obtain a Jordan curve $\gamma$ and by the Jordan Curve Theorem $\gamma$ is the boundary of a bounded domain int $\gamma$. Now let $\Omega$ be the open set equal to $C F \cap$ int $\gamma$. Since $x y \subset C F$ it follows that $\overline{x y} \subset \partial \Omega$, therefore $\overline{x y}$ is contained in the convex hull $T$ of $\bar{\Omega}$ and consequently no point of $x y$ is an extreme point of $T$. The Krein-Milman Theorem (see [2, p. 1]) says that $T$ is the convex hull of its extreme points, so $T$ has at least 3 extreme points and hence $T$ has an extreme point which does not lie on $\overline{x y}$. Without loss of generality this point may be taken as the origin 0 and we may suppose (since $T$ is convex) that $T \subset \bar{\Omega}_{0}$ where $\Omega_{0}=\left\{(\xi, \eta) \in \mathbb{R}^{2}: \eta>0\right\}$. Since 0 is an extreme point of $T$ it follows that $0 \in \partial \Omega$. Now $d_{\overline{x y}}(0)>0$ so $0 \in F$ and therefore $d_{C D}(0)>0$, hence we may suppose without loss of generality that $\min \left\{d_{C D}(0), d_{\overline{x y}}(0) / 2\right\}=1$. Now

$$
d_{C \Omega}=\min \left\{d_{\gamma}, d_{F}\right\}=\min \left\{d_{\overline{x y}}, d_{F}\right\}
$$

in $\Omega$, so $d_{C \Omega}=d_{F}$ in $B(0,1) \cap \Omega$ and hence $d_{C \Omega}$ is subharmonic in $B(0,1)$. By Lemma B every point in $B(0,1)$ has a unique nearest point in $C \Omega$, so by Theorem 1 , $B((0,1), 1) \subset \Omega$. Now let $L_{1}$ and $L_{2}$ be the sets $\{(\xi, 0): \xi>0\}$ and $\{(\xi, 0): \xi<0\}$ respectively. The extremity of 0 with respect to $T$ implies that either $L_{1} \cap T$ or $L_{2} \cap T$ is empty. Without loss of generality we may suppose that $L_{1} \cap T$ is empty. This gives that $d_{C \Omega}<h$ in $\{(\xi, \eta): \xi>0, \eta>0\}$ where $h: \mathbb{R}^{2} \rightarrow \mathbb{R}$ is given by $h((\xi, \eta))=\eta\left((\xi, \eta) \in \mathbb{R}^{2}\right)$. Further $d_{C \Omega} \leq h$ in $\mathbb{R}^{2}$ and so

$$
\begin{aligned}
d_{F}\left(\left(0, \frac{1}{2}\right)\right) & =d_{C \Omega}\left(\left(0, \frac{1}{2}\right)\right)=\frac{1}{2}=h\left(\left(0, \frac{1}{2}\right)\right)=\mathcal{M}\left(h,\left(0, \frac{1}{2}\right), \frac{1}{4}\right) \\
& >\mathcal{M}\left(d_{C \Omega},\left(0, \frac{1}{2}\right), \frac{1}{4}\right)=\mathcal{M}\left(d_{F},\left(0, \frac{1}{2}\right), \frac{1}{4}\right),
\end{aligned}
$$

a contradiction.

5. A counterexample for higher dimensions. In this section we show that Theorem 2 does not hold for $N \geq 3$.

Let $F$ be the circumference $\left\{x=\left(x_{1}, x_{2}, \ldots, x_{N}\right) \in \mathbb{R}^{N}: x_{1}^{2}+x_{2}^{2}=1, x_{3}=x_{4}=\right.$ $\left.\cdots=x_{N}=0\right\}$ and let $\rho$ denote $\sqrt{x_{1}^{2}+x_{2}^{2}}$. Then

$$
d_{F}(x)=\sqrt{(\rho-1)^{2}+x_{3}^{2}+\cdots+x_{N}^{2}}
$$

and so

$$
\Delta d_{F}(x)=\frac{N-1-1 / \rho}{\sqrt{(\rho-1)^{2}+x_{3}^{2}+\cdots+x_{N}^{2}}} \quad(\rho \neq 0) .
$$

Therefore $d_{F}$ is subharmonic in $D=\left\{\left(x_{1}, x_{2}, \ldots, x_{N}\right) \in \mathbb{R}^{N}: x_{1}^{2}+x_{2}^{2}>(N-1)^{-2}\right\}$ which contains $F$. Since $F$ is not convex, we have shown that Theorem 2 fails in higher dimensions. 


\section{REFERENCES}

1. D. H. Armitage and Ü. Kuran, The convexity of a domain and the superharmonicity of the signed distance function, Proc. Amer. Math. Soc. 93 (1985), 598-600.

2. R. R. Phelps, Lectures on Choquet's theorem, Van Nostrand, Princeton, N.J., 1966.

3. F. A. Valentine, Convex sets, McGraw-Hill, New York, 1964.

4. S. Willard, General topology, Addison-Wesley, Reading, Mass., 1970.

Department of Pure Mathematics, Liverpool University, Liverpool, EngLAND

Current address: 195 Pitville Avenue, Liverpool, L18 7JH, England 